\title{
DISTRIBUTIVE LATTICES HAVING $n$-PERMUTABLE CONGRUENCES
}

\author{
M. E. ADAMS AND R. BEAZER
}

(Communicated by Louis J. Ratliff, Jr.)

\begin{abstract}
Distributive lattices having $n$-permutable congruences are characterized by the property that they have no $n$-element chain in their poset of prime ideals.
\end{abstract}

\section{INTRODUCTION}

During the course of investigations into various congruence properties of distributive lattices, $p$-algebras, double $p$-algebras, and de Morgan algebras in $[1,2,5]$, we are led to consider, for integers $n$ with $1 \leq n \leq 4$, the class $\mathscr{D}_{n}$ of all distributive lattices having no $(n+1)$-element chain in their poset of prime ideals. In [2], the members of $\mathscr{D}_{n}$, for any $n \geq 1$, are characterized by a sentence in the first-order theory of distributive lattices (see Proposition 3.1). $\mathscr{D}_{n}$ contains the lattice reducts of the members of several important varieties of algebras arising in the study of many-valued logics; including the variety of Lukasiewicz algebras of order $n+1$ and the variety $\mathscr{L}_{n+1}$ of $L$-algebras generated by the $(n+1)$-element chain algebra (see [3, 4]). Earlier research shows that those distributive lattices, $p$-algebras, and double $p$-algebras having permutable congruences are precisely the ones whose lattice reduct belongs to $\mathscr{D}_{1}, \mathscr{D}_{2}$, and $\mathscr{D}_{3}$, respectively (see $\left.[2,5]\right)$. Furthermore, those distributive lattices, $p$-algebras, double $p$-algebras, and de Morgan algebras whose compact congruences are all principal are precisely the ones whose lattice reduct belongs to $\mathscr{D}_{2}, \mathscr{D}_{3}, \mathscr{D}_{4}$, and $\mathscr{D}_{4}$, respectively (see $\left.[1,2,5]\right)$. It is natural to ask if the members of $\mathscr{D}_{n}$ can be characterized by some property of their congruences. In this note we answer this question affirmatively by showing that $\mathscr{D}_{n}$ is the class of all distributive lattices having $(n+1)$-permutable congruences.

\section{Preliminaries}

Henceforth, $n$ will denote an arbitrary integer $\geq 1$. If $\theta, \psi$ are binary relations on a set $L$ then $\theta \circ \psi$ will denote their relational product and $\theta \circ^{n} \psi$ will stand for the compound relational product $\theta \circ \psi \circ \theta \circ \cdots$, involving $n$ factors, starting with $\theta$ and thereafter alternating between $\theta$ and $\psi$. In the event that

Received by the editors May 1, 1990.

1980 Mathematics Subject Classification (1985 Revision). Primary 06D05, 06B10. 
$n \geq 2$ and $\theta \circ^{n} \psi=\psi \circ^{n} \theta$ we will say that $\theta$ and $\psi n$-permute (or are $n$ permutable). If $\theta$ and $\psi$ 2-permute we will simply say that they permute (or are permutable). An algebra is said to have n-permutable congruences if every pair of congruences on it $n$-permute.

If $L$ is a lattice $a, b, c, d \in L, b \leq a$ and $d \leq c$ then we will indicate that the quotient $a / b$ is perspective to the quotient $c / d$ by writing $a / b \sim c / d$ and denote by $\theta(a, b)$ the principal congruence on $L$ collapsing $a / b$.

For all other notation and terminology, we refer the reader to [6].

\section{The Class $\mathscr{D}_{n}$}

The following was proved in [2]:

Proposition 3.1. A distributive lattice $L$ belongs to $\mathscr{D}_{n}$ iff, given any $x_{i} \in L$ with $0 \leq i \leq n+1$ and $x_{0} \leq x_{1} \leq \cdots \leq x_{n+1}$, there exist $x_{i}^{\prime} \in L$ for $1 \leq i \leq n$ such that

$$
x_{0}=x_{1} \wedge x_{1}^{\prime}, x_{i} \vee x_{i}^{\prime}=x_{i+1} \wedge x_{i+1}^{\prime}
$$

when $1 \leq i<n$ and $x_{n} \vee x_{n}^{\prime}=x_{n+1}$.

In order to pave the way for our new characterization of the members of $\mathscr{D}_{n}$ we first prove the following:

Lemma 3.2. Congruences $\theta$ and $\psi$ of a lattice $(n+1)$-permute iff the binary relations $\theta \cap \leq$ and $\psi \cap \leq(n+1)$-permute.

Proof. Throughout, let

$$
\alpha=\left\{\begin{array}{ll}
\theta, & \text { if } n \text { is even } \\
\psi, & \text { if } n \text { is odd }
\end{array} \text { and } \alpha^{\prime} \in\{\theta, \psi\} \backslash\{\alpha\} .\right.
$$

Suppose that $\theta$ and $\psi(n+1)$-permute and let $(x, y) \in(\theta \cap \leq) \circ^{n+1}(\psi \cap \leq)$. Then there exist $x_{i} \in L, 1 \leq i \leq n$, such that $x \leq x_{1} \leq \cdots \leq x_{n} \leq y$ and $x \equiv x_{1}(\theta), x_{1} \equiv x_{2}(\psi), \ldots, x_{n} \equiv y(\alpha)$. Therefore there exist $x_{i}^{\prime} \in L$, $1 \leq i \leq n$, such that

$$
x \equiv x_{1}^{\prime}(\psi), x_{1}^{\prime} \equiv x_{2}^{\prime}(\theta), \ldots, x_{n}^{\prime} \equiv y\left(\alpha^{\prime}\right) .
$$

For $1 \leq i \leq n$, define $\bar{x}_{i} \in[x, y]$ by $\bar{x}_{i}=x \vee\left(x_{1}^{\prime} \wedge y\right)$. Clearly, $x \equiv \bar{x}_{1}(\psi)$, $\bar{x}_{1} \equiv \bar{x}_{2}(\theta), \ldots, \bar{x}_{n} \equiv y\left(\alpha^{\prime}\right)$. Now define elements $X_{i}^{\prime} \in L$, for $1 \leq i \leq n$, by $X_{1}^{\prime}=\bar{x}_{1}$ and $X_{k+1}^{\prime}=X_{k}^{\prime} \vee \bar{x}_{k+1}$ whenever $1 \leq k<n$. Clearly

$$
x \leq X_{1}^{\prime} \leq X_{2}^{\prime} \leq \cdots \leq X_{n}^{\prime} \leq y
$$

and $x \equiv X_{1}^{\prime}(\psi), X_{1}^{\prime} \equiv X_{2}^{\prime}(\theta), \ldots, X_{n}^{\prime} \equiv y\left(\alpha^{\prime}\right)$. Therefore $(x, y) \in$ $(\psi \cap \leq) \circ^{n+1}(\theta \cap \leq)$. Similarly, $(\psi \cap \leq) \circ^{n+1}(\theta \cap \leq) \subseteq(\theta \cap \leq) \circ^{n+1}(\psi \cap \leq)$.

Suppose now that $\theta \cap \leq$ and $\psi \cap \leq(n+1)$-permute. Let $(x, y) \in \theta \circ^{n+1} \psi$. Then there exist $x_{i} \in L, 1 \leq i \leq n$, such that $x \equiv x_{1}(\theta), x_{1} \equiv x_{2}(\psi), \ldots, x_{n}$ $\equiv y(\alpha)$. Define elements $X_{i} \in L, 1 \leq i \leq n$, by $X_{1}=x \vee x_{1}$ and $X_{k+1}=$ $X_{k} \vee x_{k+1}$, whenever $1 \leq k<n$, and let $Y=X_{n} \vee y$. Clearly,

$$
x \leq X_{1} \leq X_{2} \leq \cdots \leq X_{n} \leq Y
$$


and $x \equiv X_{1}(\theta), X_{1} \equiv X_{2}(\psi), \ldots, X_{n} \equiv Y(\alpha)$; in other words, $(x, Y) \in$ $(\theta \cap \leq) o^{n+1}(\psi \cap \leq)$. Therefore $(x, Y) \in(\psi \cap \leq) \circ^{n+1}(\theta \cap \leq)$ and so there exist $x_{i}^{\prime} \in L, 1 \leq i \leq n$, such that

$$
x \leq x_{1}^{\prime} \leq x_{2}^{\prime} \leq \cdots \leq x_{n}^{\prime} \leq Y
$$

and $x \equiv x_{1}^{\prime}(\psi), x_{1}^{\prime} \equiv x_{2}^{\prime}(\theta), \ldots, x_{n}^{\prime} \equiv Y\left(\alpha^{\prime}\right)$. Now define elements $Y_{i} \in L$, $1 \leq i \leq n$, by $Y_{1}=x_{n} \vee y$ and $Y_{k+1}=x_{n-k} \vee Y_{k}$ whenever $1 \leq k<n$. Observe that $Y=x \vee Y_{n}$,

$$
y \leq Y_{1} \leq Y_{2} \leq \cdots \leq Y_{n} \leq Y
$$

and $y \equiv Y_{1}(\alpha), \ldots, Y_{n-1} \equiv Y_{n}(\psi), Y_{n} \equiv Y(\theta)$. Therefore there exist $y_{i}^{\prime} \in L$, $1 \leq i \leq n$, such that

$$
y \leq y_{1}^{\prime} \leq \cdots \leq y_{n-1}^{\prime} \leq y_{n}^{\prime} \leq Y
$$

and

$$
y \equiv y_{1}^{\prime}\left(\alpha^{\prime}\right), \ldots, y_{n-1}^{\prime} \equiv y_{n}^{\prime}(\theta), \quad y_{n}^{\prime} \equiv Y(\psi) \text {. }
$$

Now,

$$
\begin{aligned}
& x=x \wedge Y \equiv x_{1}^{\prime} \wedge y_{n}^{\prime}(\psi), \\
& x_{1}^{\prime} \wedge y_{n}^{\prime} \equiv x_{2}^{\prime} \wedge y_{n-1}^{\prime}(\theta), \\
& x_{2}^{\prime} \wedge y_{n-1}^{\prime} \equiv x_{3}^{\prime} \wedge y_{n-2}^{\prime}(\psi) \text {, } \\
& x_{n-1}^{\prime} \wedge y_{2}^{\prime} \equiv x_{n}^{\prime} \wedge y_{1}^{\prime}(\alpha), \quad \text { and } \\
& x_{n}^{\prime} \wedge y_{1}^{\prime} \equiv Y \wedge y\left(\alpha^{\prime}\right)=y \text {. }
\end{aligned}
$$

Thus $(x, y) \in \psi \circ^{n+1} \theta$. Similarly, we can show that $\psi \circ^{n+1} \theta \subseteq \theta \circ^{n+1} \psi$ and so $\theta$ and $\psi(n+1)$-permute.

A well-known consequence of Proposition 3.1 is that a distributive lattice belongs to $\mathscr{D}_{1}$ iff it is relatively complemented. It is also known that a distributive lattice has permutable congruences iff it is relatively complemented. This yields the case where $n=1$ in the following:

Theorem 3.3. Let $L$ be a distributive lattice. Then $L \in \mathscr{D}_{n}$ iff it has $(n+1)$ permutable congruences.

Proof. Suppose that $n \geq 2$ and $L \in \mathscr{D}_{n}$. Let $\theta, \psi$ be congruences of $L$ and $(x, y) \in(\theta \cap \leq) \circ^{n+1}(\psi \cap \leq)$. For the sake of notational convenience, let us suppose that $n$ is odd. Then there exist $x_{i} \in L$ for $1 \leq i \leq n$ such that

$$
x=x_{0} \leq x_{1} \leq \cdots \leq x_{n} \leq x_{n+1}=y
$$

and

$$
x_{0} \equiv x_{1}(\theta), x_{1} \equiv x_{2}(\psi), \ldots, x_{n} \equiv x_{n+1}(\psi) .
$$

By Proposition 3.1, there are $x_{i}^{\prime} \in L$ for $1 \leq i \leq n$ such that $x=x_{0}=x_{1} \wedge x_{1}^{\prime}$, $x_{i} \vee x_{i}^{\prime}=x_{i+1} \wedge x_{i+1}^{\prime}$, for $1 \leq i \leq n$, and $x_{n} \vee x_{n}^{\prime}=x_{n+1}=y$. Let us write 
$z_{0}=x$ and $z_{i}=x_{i} \vee x_{i}^{\prime}$ when $1 \leq i \leq n$. Observe that $z_{1} / x_{1} \sim x_{1}^{\prime} / x$ and $x_{1} \equiv z_{1}(\psi)$, so that $x \equiv x_{1}^{\prime}(\psi)$, and furthermore, $x_{n} / z_{n-1} \sim y / x_{n}^{\prime}$ and $z_{n-1} \equiv x_{n}(\theta)$, so that $x_{n}^{\prime} \equiv y(\theta)$. Suppose now that $i$ is odd and $3 \leq i \leq n$. Then $x_{i-1} / z_{i-2} \sim z_{i-1} / x_{i-1}^{\prime}$ and $z_{i-2} \equiv x_{i-1}(\psi)$ so that $x_{i-1}^{\prime} \equiv z_{i-1}(\psi)$, and furthermore $z_{i} / x_{i} \sim x_{i}^{\prime} / z_{i-1}$ and $x_{i} \equiv z_{i}(\psi)$ so that $z_{i-1} \equiv x_{i}^{\prime}(\psi)$. Therefore $x_{i-1}^{\prime} \equiv x_{i}^{\prime}(\psi)$. It follows that

$$
x \equiv x_{1}^{\prime}(\psi), x_{2}^{\prime} \equiv x_{3}^{\prime}(\psi), \ldots, x_{n-1}^{\prime} \equiv x_{n}^{\prime}(\psi) .
$$

In the event that $i$ is even and $2 \leq i \leq n-1$, a similar argument yields $x_{i-1}^{\prime} \equiv x_{i}^{\prime}(\theta)$. Consequently,

$$
x_{1}^{\prime} \equiv x_{2}^{\prime}(\theta), x_{3}^{\prime} \equiv x_{4}^{\prime}(\theta), \ldots, x_{n}^{\prime} \equiv y(\theta) .
$$

Thus, $(x, y) \in(\psi \cap \leq) \circ^{n+1}(\theta \cap \leq)$ under the assumption that $n$ is odd. The case where $n$ is even is dealt with similarly and so, for any $n \geq 2$ and arbitrary congruences $\theta, \psi$ on $L,(\theta \cap \leq) 0^{n+1}(\psi \cap \leq) \subseteq(\psi \cap \leq) \circ^{n+1}(\theta \cap \leq)$. Therefore, by Lemma 3.2, $L$ has $(n+1)$-permutable congruences.

Suppose now that $L$ has $(n+1)$-permutable congruences. For $0 \leq i \leq n+1$, let $x_{i} \in L$ satisfy $x_{0} \leq x_{1} \leq \cdots \leq x_{n} \leq x_{n+1}$. Initially, let us suppose that $n$ is odd and so $n=2 k+1$, for some $k \geq 1$. Define congruences $\theta$ and $\psi$ of $L$ by

$$
\theta=\bigvee_{i=0}^{k} \theta\left(x_{2 i}, x_{2 i+1}\right) \text { and } \psi=\bigvee_{j=0}^{k} \theta\left(x_{2 j+1}, x_{2 j+2}\right)
$$

First, observe that $\theta \wedge \psi=\bigvee_{i, j=0}^{k} \theta\left(x_{2 i}, x_{2 i+1}\right) \wedge \theta\left(x_{2 j+1}, x_{2 j+2}\right)$, since the congruence lattice of $L$ is distributive, that $x_{2 i} \leq x_{2 i+1} \leq x_{2 j+1} \leq x_{2 j+2}$ when $i \leq j$, and that $x_{2 j+1} \leq x_{2 j+2} \leq x_{2 i} \leq x_{2 i+1}$ when $i>j$. However, it is easy to see, using the well-known description of principal congruences of distributive lattices, that if $a, b, c, d \in L$ and $a \leq b \leq c \leq d$ then $\theta(a, b) \wedge \theta(c, d)=\omega$. Therefore $\theta \wedge \psi=\omega$. Now, $x_{0} \equiv x_{1}(\theta), x_{1} \equiv x_{2}(\psi), \ldots, x_{n} \equiv x_{n+1}(\psi)$ and so there exist $x_{i}^{\prime} \in L$, for $1 \leq i \leq n$, such that

$$
x_{0} \leq x_{1}^{\prime} \leq \cdots \leq x_{n}^{\prime} \leq x_{n+1}
$$

and $x_{0} \equiv x_{1}^{\prime}(\psi), x_{1}^{\prime} \equiv x_{2}^{\prime}(\theta), \ldots, x_{n}^{\prime} \equiv x_{n+1}(\theta)$, by Lemma 3.2. Let us define, for $1 \leq i \leq n, X_{i}^{\prime}=\left(x_{i-1} \vee x_{i}^{\prime}\right) \wedge x_{i+1}$ and note that $X_{i}^{\prime} \in\left[x_{i-1}, x_{i+1}\right]$.

We claim that $x_{1} \wedge X_{1}^{\prime}=x_{0}$. Indeed, $x_{1} \wedge X_{1}^{\prime}=x_{1} \wedge\left[\left(x_{0} \vee x_{1}^{\prime}\right) \wedge x_{2}\right]=\left(x_{0} \vee x_{1}^{\prime}\right) \wedge$ $x_{1}=x_{1}^{\prime} \wedge x_{1}$ so that $x_{1}^{\prime} \wedge X_{1}^{\prime} \equiv x_{1}^{\prime} \wedge x_{0}(\theta)=x_{0}$ and $x_{1} \wedge X_{1}^{\prime} \equiv x_{0} \wedge x_{1}(\psi)=x_{0}$. Therefore $x_{1} \wedge X_{1}^{\prime} \equiv x_{0}(\theta \wedge \psi)$ and so $x_{1} \wedge X_{1}^{\prime}=x_{0}$.

Next we show that $x_{n} \vee X_{n}^{\prime}=x_{n+1}$. Indeed, $x_{n} \vee X_{n}^{\prime}=x_{n} \vee\left[\left(x_{n-1} \vee x_{n}^{\prime}\right) \wedge\right.$ $\left.x_{n+1}\right]=x_{n} \vee x_{n}^{\prime}$ so $x_{n} \vee X_{n}^{\prime} \equiv x_{n+1} \vee x_{n}^{\prime}(\psi)=x_{n+1}$ and $x_{n} \vee X_{n}^{\prime} \equiv x_{n} \vee x_{n+1}(\theta)=$ $x_{n+1}$. Therefore $x_{n} \vee X_{n}^{\prime} \equiv x_{n+1}(\theta \wedge \psi)$ and so $x_{n} \vee X_{n}^{\prime}=x_{n+1}$.

It remains to show that $x_{i} \vee X_{i}^{\prime}=x_{i+1} \wedge X_{i+1}^{\prime}$, for $1 \leq i<n$. Observe that, for $1 \leq i<n, x_{i} \vee X_{i}^{\prime}=x_{i} \vee\left[\left(x_{i-1} \vee x_{i}^{\prime}\right) \wedge x_{i+1}\right]=\left(x_{i} \vee x_{i}^{\prime}\right) \wedge x_{i+1}$ 
and suppose that $i$ is odd. Then $x_{i} \equiv x_{i+1}^{\prime}(\psi)$ and $x_{i} \equiv x_{i+1}^{\prime}(\theta)$. Therefore, $x_{i} \vee X_{i}^{\prime} \equiv\left(x_{i} \vee x_{i+1}^{\prime}\right) \wedge x_{i+1}(\theta)=x_{i+1} \wedge\left[\left(x_{i} \vee x_{i+1}^{\prime}\right) \wedge x_{i+2}\right]=x_{i+1} \wedge X_{i+1}^{\prime}$; that is, $x_{i} \vee X_{i}^{\prime} \equiv x_{i+1} \wedge X_{i+1}^{\prime}(\theta)$. Furthermore, $x_{i} \vee X_{i}^{\prime} \equiv\left(x_{i} \vee x_{i}^{\prime}\right) \wedge x_{i}(\psi)=x_{i}$ and $x_{i+1} \wedge X_{i+1}^{\prime}=x_{i+1} \wedge\left[\left(x_{i} \vee x_{i+1}\right) \wedge x_{i+2}\right]=x_{i+1} \wedge\left(x_{i}^{\prime} \vee x_{i+1}\right) \equiv x_{i} \wedge\left(x_{i} \vee x_{i+1}^{\prime}\right)(\psi)=x_{i}$ so $x_{i} \vee X_{i}^{\prime} \equiv x_{i+1} \wedge X_{i+1}^{\prime}(\psi)$. Therefore, $x_{i} \vee X_{i}^{\prime} \equiv x_{i+1} \wedge X_{i+1}^{\prime}(\theta \wedge \psi)$ and so $x_{i} \vee X_{i}^{\prime}=x_{i+1} \wedge X_{i+1}^{\prime}$. When $i$ is even and $1 \leq i<n$ we have $x_{i} \equiv x_{i+1}(\theta)$ and $x_{i}^{\prime} \equiv x_{i+1}^{\prime}(\psi)$ and an argument similar to the one where $i$ is odd also yields $x_{i} \vee X_{i}^{\prime} \equiv x_{i+1} \wedge X_{i+1}^{\prime}(\theta \wedge \psi)$. Thus, $x_{i} \vee X_{i}^{\prime}=x_{i+1} \wedge X_{i+1}^{\prime}$, for any $i$ with $1 \leq i<n$.

In the event that $n$ is even, so that $n=2 k$ for some $k \geq 1$, we define

$$
\theta=\bigvee_{i=0}^{k} \theta\left(x_{2 i}, x_{2 i+1}\right) \text { and } \psi=\bigvee_{j=1}^{k} \theta\left(x_{2 j-1}, x_{2 j}\right)
$$

Again we can show that $\theta \wedge \psi=\omega$, proceeding in a manner similar to that for which $n$ is odd and draw the same conclusions. In any case, $L \in \mathscr{D}_{n}$ by Proposition 3.1.

Corollary 3.4. The length of the poset of prime ideals of a distributive lattice is $n$ iff $L$ has $(n+2)$-permutable but not $(n+1)$-permutable congruences.

Corollary 3.5. Every compact congruence of a distributive lattice is principal iff it has 3-permutable congruences.

\section{REFERENCES}

1. M. E. Adams and R. Beazer, Congruence relations on de Morgan algebras, Algebra Universalis 26 (1989), 103-125.

2. _ Congruence properties of distributive double p-algebras, Czech. Math. J. (to appear).

3. R. Balbes and P. Dwinger, Distributive lattices, Univ. of Missouri Press, Columbia, Missouri, 1974.

4. R. Beazer, Hierarchies of distributive lattices satisfying annihilator conditions, J. London Math. Soc. 11 (1975), 216-222.

5. __ Principal congruence properties of some algebras with pseudocomplementation, Portugal. Math. (to appear).

6. G. Grätzer, General lattice theory, Birkhäuser, Basel and Stuttgart, 1978. 12561

Department of Mathematics, State University of New York, New Paltz, New York

Department of Mathematics, University of Glasgow, Glasgow, Scotland G12 8QW 